\title{
Contraste de una estructura trifactorial para escala de actitudes hacia lesbianas y hombres homosexuales (AHLG)
}

Contrast of a 3-factor structure for the Attitude Toward Lesbian and Gay Men (ATLG) scale

José Moral de la Rubia

Universidad Autónoma de Nuevo León

\author{
Adrián Valle de la $\mathrm{O}$ \\ Instituto Tecnológico y de Estudios \\ Superiores de Monterrey (ITESM)
}

\section{Resumen}

Este artículo tiene como objetivos contrastar el modelo tridimensional de Moral y Valle (2011), para la escala ATLG y aportar pruebas de su validez. Se aplicaron las escalas ATLG (Herek, 1984), de homonegatividad internalizada (HNI-16; Moral \& Valle, 2013) y homofobia (HFK-10; Klamen, Grossman, \& Kopacz, 1999) a una muestra no probabilística de 231 estudiantes mexicanos de Ciencias de la Salud (121 mujeres y 103 hombres). Se contrastaron tres modelos por Mínimos Cuadrados Generalizados (GLS): de un factor, dos factores correlacionados y tres factores jerarquizados a uno general. Los modelos de dos factores correlacionados y de tres factores jerarquizados tuvieron una bondad de ajuste equivalente y significativamente mayor que la del modelo unidimensional. No obstante, los datos reflejaron unidimensionalidad con base en tres argumentos: 1) los valores de correlación y coeficientes de regresión de los factores de primer orden fueron muy altos; 2) el análisis paralelo de Horn indicó que el número de factores es uno, por lo que la varianza explicada por un segundo o tercer factor se debería al azar; y 3) la bondad de ajuste del modelo unidimensional fue aceptable y muy semejante a la de los otros dos modelos. Las correlaciones de la puntuación total de la ATLG con las de HNI-16 y HFK-10 fueron altas y significativas. La orientación sexual autodefinida y tener amigos homosexuales tuvieron un efecto mediano sobre la ATLG. Se concluye que la ATLG es unidimensional y posee evidencias de validez. Se sugiere una forma simplificada de 5 ítems.

Palabras Clave: actitud, homosexualidad, homofobia, homonegatividad, heterosexismo.

Nota de Autor:

José Moral de la Rubia, Facultad de Psicología, Universidad Autónoma de Nuevo León; Adrián Valle de la O, Departamento de Ciencias Básicas de la Escuela de Medicina y Ciencias de la Salud, Instituto Tecnológico y de Estudios Superiores de Monterrey (ITESM).

La correspondencia en relación con este artículo debe dirigirse a José Moral de la Rubia, Facultad de Psicología, Universidad Autónoma de Nuevo León, c/Dr. Carlos Canseco 110, colonia Mitras centro, C.P. 64460, Monterrey, Nuevo León, México. 


\section{Abstract}

The aims of this paper were to compare the 3-factor model proposed by Moral and Valle (2011), for the ATLG scale, and provide validity evidences for it. The ATLG scale (Herek, 1984), the internalized homonegativity scale (HNI-16; Moral \& Valle, 2013) and the homophobia scale (HFK10; Klamen, Grossman, \& Kopacz, 1999) were applied to a non-probabilistic sample of 231 Mexican students of health sciences (121 women and 103 men). Three structural models were contrasted by Generalized Least Squares (GLS): one general factor, two correlated factors and three factors hierarchized to a general factor. The models of two correlated factors and three hierarchized factors had an equivalent goodness of fit and were significantly higher than the one-factor model. However, the data showed unidimensionality based on three arguments: 1) the values of correlations and regression coefficients of first-order factors were very high, 2) Horn's parallel analysis indicated that the number of factors is one, and thus the variance explained by a second or third factor could be due to random, and 3) the goodness of fit of the unidimensional model was acceptable and very similar to that of the other two models. The correlations of the ATLG total score with the total scores of $\mathrm{HNI}-16$ and HFK-10 were high. The self-defined sexual orientation and having gay friends had a medium size effect on the ATLG total score. It is concluded that the ATLG scale is unidimensional, and shows validity evidences. A simplified form of 5 items is suggested.

Keywords: attitude, homosexuality, homophobia, homonegativity, heterosexism.

El concepto de actitud hace referencia al posicionamiento valorativo de un individuo frente a un objeto ante el cual caben posturas que van de la aceptación total al rechazo completo, pasando por aceptaciones o rechazos parciales (Haddock, 2004).

Se suelen emplear calificativos, atribuciones y evaluaciones muy negativos y estereotipados cuando existe una actitud de rechazo hacia un grupo de individuos por poseer cierta característica, actitud que lo devalúa y descalifica, denominada estigmatización (Major \& Eccleston, 2005). El estigma usualmente se asienta en una ideología. Si esta ideología es asumida por un individuo que posee la característica objeto de estigmatización y éste muestra un comportamiento de autodesprecio, se habla de autoestigma (Herek, Chopp, \& Strohl, 2007).

La discriminación es un trato diferencial que niega, restringe o quita los beneficios, apoyos u oportunidades a los que un grupo de individuos tiene derecho; se basa en distinciones arbitrarias, injustas o injustificables (razón de discriminación) desde los marcos de valores y principios compartidos por las personas que interactúan (Moral \& Segovia, 2011).

La homosexualidad u orientación sexual hacia personas del mismo sexo ha sido objeto de estigmatización y discriminación en la sociedad occidental, sobre todo a raíz de la imposición de los valores judeocristianos de las postrimerías del Imperio Romano de Occidente (Crompton, 2006). Si en el pasado existió una criminalización de la homosexualidad en los países occidentales, actualmente el rechazo ha pasado a ser más sutil desde una ideología que sustenta la hegemonía de la heterosexualidad (Herek, 2004; Majied, 2008).

A la actitud de rechazo extremo hacia la homosexualidad junto con miedo u odio hacia las personas con orientación homosexual se le denomina homofobia (Herek, 2009). Si esta actitud extrema va dirigida hacia el otro distinto de mí, se habla de homofobia externalizada; si va 
dirigida hacia el propio deseo homosexual, se habla de homofobia internalizada. Cuando una persona presenta un fuerte deseo homosexual por determinantes biológicos o ambientales y vive en una sociedad homofóbica, el desarrollo de una orientación e identidad homosexuales conlleva un proceso de enfrentamiento contra valores asumidos, prejuicios sociales y conductas discriminatorias (Moral, 2010). En este proceso no todos los individuos lograrán asumir una identidad positiva y vivir una sexualidad integrada (Weber-Gilmore, Rose, \& Rubinstein, 2011). Esta dificultad es debida a una autoestigmatización (homofobia internalizada), así como a la persecución y control sociales (Herek, 2009; Herek, Gillis, \& Cogan, 2009).

Desde su publicación en la década de los ochenta, la escala ATLG ha sido el instrumento más usado para evaluar la actitud hacia la homosexualidad en los estudios publicados. La escala ATLG está integrada por dos subescalas, una para evaluar la actitud hacia hombres homosexuales y otra hacia lesbianas. Cada subescala es unidimensional y tiene consistencia interna alta $(\alpha \geq .85)$. La correlación entre ellas es moderada o alta (Herek \& McLemore, 2011).

En población anglófona, Stoever y Morera (2007), tras contrastar cuatro modelos estructurales para la ATLG, concluyen que el jerarquizado con un factor general de prejuicio sexual presenta el mejor ajuste a los datos. Blackwell y Kiehl (2008) obtuvieron evidencia de unidimensionalidad por análisis factorial confirmatorio para la variable latente de rechazo hacia la homosexualidad con 16 de los 20 ítems de la ATLG. Chonody, Siebert, Siebert y Rutledget (2011), también defienden un modelo unidimensional frente al de dos factores correlacionados para la versión abreviada de 10 ítems de la ATLG propuesta por Herek (1994).

La escala ATLG ha sido estudiada en países latinos. Cárdenas y Barrientos (2008a), en una muestra de 142 estudiantes chilenos de Psi- cología y Economía, por análisis de componentes principales, obtuvieron 3 factores correlacionados que explicaban $61 \%$ de la varianza total para los 10 ítems de actitud hacia las lesbianas: valores tradicionales (L3, L5, L6, L8, L9 y L10), sanción social ( $L 4$ y L7) y derechos sociales (L1 y L7); y dos factores correlacionados que explicaban 54\% de la varianza total para los 10 ítems de actitud hacia los hombres homosexuales: derechos y estereotipos (G1, G2, G3, G4, G6, G9 y G10) y natural/antinatural (G5, G7 y G8). Barrientos y Cárdenas (2012) hallaron un ajuste superior de un modelo de 5 factores de primer orden subordinados a dos factores de segundo orden correlacionados en comparación con el unidimensional y de dos factores correlacionados, reflejando sus índices un ajuste aceptable en términos generales $\left(\chi^{2} / \mathrm{gl}=3.34, \mathrm{CFI}=.93\right.$, $\mathrm{NFI}=.91, \mathrm{RFI}=.88$ y RMSEA $=.06)$ por Máxima Verosimilitud.

Moral y Valle (2011), en una muestra de 356 estudiantes mexicanos de Ciencias de la Salud, encontraron una consistencia interna alta para los 20 ítems de la ATLG $(\alpha=.94)$. Por análisis de componentes principales con rotación Oblimin, fijando el número de factores por el criterio de Kaiser, obtuvieron un factor de rechazo hacia las lesbianas $(\alpha=.91)$ y dos factores de rechazo abierto (G2, G3, G4, G6 y G10) ( $\alpha=$ .85) y sutil (G1, G5, G7, G8 y G9) ( $\alpha=.78$ ) hacia los hombres homosexuales. Las correlaciones entre los 3 factores fueron moderadas, de .39 a .55, en el análisis factorial exploratorio. Esta estructura de tres factores correlacionados tuvo un ajuste aceptable a los datos en términos generales $\left(\chi^{2} / \mathrm{gl}=2.11, \mathrm{FD}=0.99, \mathrm{PNCP}=\right.$ $0.52, \mathrm{GFI}=.90, \mathrm{AGFI}=.88$ y RMSEA $=.06$ ) por GLS. El modelo sin restricciones mostró propiedades aceptables de invarianza entre ambos $\operatorname{sexos}\left(\chi^{2} / g l=1.72, \mathrm{FD}=1.62, \mathrm{PNCP}=0.68\right.$, $\mathrm{GFI}=.84, \mathrm{AGFI}=.80$ y RMSEA $=.04)$. No obstante, las correlaciones entre los tres factores fueron de .89. Estas altas correlaciones sugieren fuertemente unidimensionalidad o subordinación de los tres factores a uno general; este 
último modelo multidimensional será el que se considerará en el presente estudio.

Entre las evidencias de validez convergente aportadas para la escala ATLG se encuentran su correlación alta con escalas de homofobia externalizada o actitud explícita hacia la homosexualidad (Moral \& Valle, 2012), homofobia internalizada (White \& Murrell, 2012) y actitud implícita (Cárdenas \& Barrientos, 2008b). Asimismo, como evidencias de validez de constructo se tiene la media de la ATLG más alta en personas que se definen heterosexuales frente a las que no en concordancia con su grupo de pertenencia o identidad (Herek \& McLemore, 2013; Turner \& Reynolds, 2007); en aquéllos que tienen amigos homosexuales frente a los que no por el contacto personal con el objeto que es estigmatizado desde el estereotipo (Herek \& McLemore, 2013); y en aquéllos que atienden a pacientes infectados de $\mathrm{VIH}$ frente a los que no también por el contacto con el objeto estigmatizado, al ser la mayoría de ellos hombres que tienen sexo con hombres (Klamen et al., 1999).

Se observa que las medias de la puntuación total de la ATLG y su factor de rechazo hacia los hombres homosexuales son mayores en los hombres que en las mujeres, debido al gran estigma cultural sobre la homosexualidad masculina y la actitud más rígida hacia la homosexualidad en el propio sexo y al servicio de una identidad heterosexual en una sociedad heterosexista. Asimismo, la media de rechazo hacia las lesbianas es equivalente o mayor en mujeres que en los hombres por la actitud más rígida hacia la homosexualidad en propio sexo, pero con menor presión de rechazo cultural (Herek \& McLemore, 2013; Moral \& Valle, 2012).

La epidemia del Virus de Inmunodeficiencia Humana (VIH), ha intensificado la estigmatización y discriminación contra los hombres con prácticas homosexuales al señalarlos como uno de los principales grupos de riesgo de infección de VIH y al hacerlos responsables de la dispersión y feminización de la epidemia (Aguirre \&
Rendón, 2008; Neumann, Hülsenbeck, \& Seibt, 2004). Existen quejas sobre discriminación dentro del sector de salud por causa de la orientación sexual, especialmente entre pacientes que viven con VIH (Córdova, Ponce, \& Valdespino, 2009). Para prevenir la violación de los derechos de estos pacientes, en algunos países como Estados Unidos de América, se están evaluando las actitudes hacia la homosexualidad e implementando talleres de cambio actitudinal en estudiantes de Ciencias de la Salud (Mayer et al., 2012; Sequeira, Chakraborti, \& Panunti, 2012).

Al ser la escala ATLG uno de los instrumentos con mejores propiedades psicométricas, empleado en diversos países y validado en México, se considera una de las mejores opciones para evaluar la actitud hacia la homosexualidad. El estudio de validación en México de Moral y Valle (2011), propuso una estructura factorial novedosa y de especial interés ante el cambio actitudinal en la cultura occidental, al distinguir entre rechazo sutil y abierto en la actitud hacia los hombres homosexuales. Esta estructura se descubrió a través de análisis factorial exploratorio; en la muestra del estudio exploratorio, el modelo de tres factores correlacionados presentó un ajuste aceptable para los datos. No obstante, las correlaciones entre los factores fueron muy altas, por lo cual se requiere una contrastación en una muestra independiente y valorar si es o no una solución artificiosa; la unidimensionalidad es el verdadero modelo subyacente.

Con base en lo anterior, la presente investigación tiene como objetivo general contrastar el modelo tridimensional propuesto por Moral y Valle (2011) para la escala ATLG, y determinar si es un modelo artificioso que no aporte utilidad adicional al modelo de un factor o al modelo original de dos factores correlacionados (Herek \& McLemore, 2011), los cuales aparecerían como hipótesis alternativas.

Dicho estudio tiene como objetivos específicos: 1) explorar la estructura factorial de la 
escala ATLG, definiendo el número de factores desde los criterios de Horn (matemático), Kaiser (pragmático) y la expectativa de tres factores (Moral \& Valle, 2011); 2) calcular la consistencia interna de la escala y sus factores; 3 ) contrastar un modelo de tres factores jerarquizados a uno general derivado de las sugerencias de Moral y Valle (2011), como hipótesis alternativas se consideran un modelo unidimensional, un modelo bidimensional (Herek \& McLemore, 2011) y algún otro posible modelo generado desde el análisis factorial exploratorio; 4) describir las distribuciones de la puntuación total de la ATLG y sus factores; 5) comparar las medias entre los factores; 6) estudiar la validez convergente en relación con la homonegatividad internalizada y la homofobia externalizada; y 7) probar la validez de constructo al comparar las medias por sexos, orientación sexual autodefinida, haber iniciado la vida sexual de pareja, tener amistades homosexuales, tener amistades infectadas de $\mathrm{VIH}$ y atender a pacientes infectados de $\mathrm{VIH}$.

Se entiende por homonegatividad internalizada la imagen, valoración y actitud negativas hacia la homosexualidad. El adjetivo "internalizada" hace referencia no sólo a su origen social y al proceso de adquisición como una función interna automática (internalización), sino también a la focalización del rechazo hacia los propios deseos y conductas (Currie, Cunningham, \& Findlay, 2004).

Se espera que: 1) el número de factores sea 3 al aplicar el criterio de Kaiser e incluso el criterio de Horn en el análisis factorial exploratorio, definiéndose los factores de rechazo sutil hacia hombres homosexuales, rechazo abierto hacia hombres homosexuales y rechazo hacia lesbianas tras una rotación oblicua (Moral \& Valle, 2011); 2) valores de consistencia interna altos (Herek \& McLemore, 2011), siendo algo menor la consistencia interna del factor de rechazo sutil hacia los hombres homosexuales en comparación con la de los factores de rechazo abierto hacia los hombres homosexuales y rechazo hacia las lesbianas (Moral \& Valle, 2011); 3) mejor ajuste del modelo de tres factores jerarquizados a un factor general en comparación con el modelo de un factor (Blackwell \& Kiehl, 2008; Chonody et al., 2011) y de dos factores correlacionados (Herek \& McLemore, 2011); 4) distribución normal, al menos en la puntuación total de la ATLG, al tratarse de un aspecto actitudinal adaptativo; 5) mayor promedio en el factor de rechazo sutil hacia los hombres homosexuales en comparación con los promedios de los factores de rechazo abierto hacia hombres homosexuales y rechazo hacia las lesbianas, por el cambio hacia una condena sutil de la homosexualidad en la sociedad actual que tradicionalmente ha condenado más la homosexualidad masculina en comparación con la femenina (Herek, 2004; Moral \& Valle, 2012); 6) mayor correlación del factor de rechazo sutil hacia los hombres homosexuales con homonegatividad internalizada, y de los factores de rechazo abierto hacia los hombres homosexuales y rechazo hacia las lesbianas con homofobia externalizada por la afinidad de contenidos (Moral \& Valle, 2012); y 7 ) menor rechazo de la homosexualidad en mujeres debido al mayor rechazo cultural hacia la homosexualidad masculina (Herek, 2004), éste se asienta en una representación del deseo sexual femenino como débil y al servicio de un deseo sexual masculino más fuerte (Wittig, 2005); menor rechazo en aquellos con orientación autodefinida no heterosexual debido a la congruencia con la orientación sexual autodefinida y defensa del grupo de identidad y pertenencia (Turner \& Reynolds, 2007); en aquellos que han iniciado su vida sexual de pareja debido a la menor necesidad de una afirmación defensiva de la orientación heterosexual ante la ideología heterosexista (Herek \& McLemore, 2013), así como en aquellos que tienen amistades homosexuales, amistades infectadas de $\mathrm{VIH}$ o han atendido a pacientes infectados de $\mathrm{VIH}$ a causa del contacto personal con el objeto estigmatizado (Herek \& McLemore, 2013). 


\section{Método}

\section{Participantes}

La población estuvo constituida por estudiantes universitarios de Ciencias de la Salud. Se obtuvo una muestra no probabilística de 231 participantes voluntarios, 100 (43\%) encuestados en la Facultad de Medicina de la Universidad Autónoma de Coahuila, 66 (29\%) en la Escuela de Medicina del Tecnológico de Monterrey y $65(28 \%)$ de la Facultad de Psicología de la Universidad Autónoma de Nuevo León. De los 224 participantes que especificaron su sexo, 121 (54\%) dijeron ser mujeres y 103 (46\%) hombres, siendo estadísticamente equivalente la frecuencia de ambos sexos (prueba binomial: $p=.26)$. La media de edad fue de 19.13 años (DE $=1.68)$, con un mínimo de 17 y máximo de 37. Respecto a la adscripción religiosa, 182 (79\%) dijeron ser católicos, 10 (4\%) cristianos y $39(17 \%)$ pertenecen a otras religiones o tienen creencias religiosas personales. Ninguno se definió como ateo o sin religión.

\section{Instrumentos}

Escala de Actitudes hacia Lesbianas y Hombres Homosexuales (ATLE; Herek, 1984). Se compone de 20 ítems tipo Likert, 10 para medir la actitud hacia los hombres homosexuales y 10 para medir la actitud hacia las lesbianas. Los ítems directos de aceptación de la homosexualidad masculina (4 ítems: G1, G5, G7 y G9) y del lesbianismo (3 ítems: L2, L4 y L7) tienen 5 opciones de respuesta y un rango de 1 a 9 (de 1, "totalmente de acuerdo," a 9, "definitivamente en desacuerdo"). La suma de éstos con los restantes 13 ítems (ítems G2, G3; G4, G6, G8, G9, L1, L3, L5, L6, L8, L9 y L10), invertidos en sus puntuaciones (de 9, "totalmente de acuerdo" a 1 "definitivamente en desacuerdo"), proporciona un puntaje que a mayor valor refleja más rechazo hacia la homosexualidad. Su rango es de 20 a 180 (Herek, 1984). En este estudio se empleó la traducción al español de Cárdenas y Barrientos (2008a).

La escala de Homonegatividad Internalizada (HNI-16) de Moral y Valle (2013). Está integrada por 16 ítems tipo Likert con 5 opciones de respuesta y un rango de 1 a 9 puntos. Todos ellos son directos. Su consistencia interna es alta $(\alpha=.88)$ y consta de 3 factores: rechazo de la manifestación pública de la homosexualidad $(\alpha=.81)$; rechazo de sentimientos, deseos e identidad homosexuales propios $(\alpha=.81)$; e incapacidad para establecer relaciones interpersonales íntimas $(\alpha=.69)$. El ajuste a los datos de un modelo de tres factores jerarquizado a uno general por GLS varía de bueno $\left(\chi^{2} / \mathrm{gl}=\right.$ 1.66; PNCP $=0.29$; y RMSEA $=.05, \mathrm{IC} 90 \%$ : $.04, .07)$ a aceptable (FD $=0.73$, GFI $=.91 \mathrm{y}$ AGFI = .88). La distribución de su puntuación total se ajusta a una curva normal (Moral \& Valle, 2013).

Escala de Homofobia (HFK) de Klamen et al. (1999). Fue diseñada ex profeso para estudiantes de Medicina, a partir de la revisión de 4 escalas publicadas de 1980 a 1993. Consta de 12 ítems tipo Likert con 4 opciones de respuestas y un rango de 1 a 7 puntos. Se eliminaron dos ítems: "me siento más negativo hacia la homosexualidad desde la epidemia del SIDA" y "la homosexualidad es un trastorno mental." El primero se descartó porque no es aplicable a los jóvenes estudiantes de hoy, pues la epidemia tiene más de 30 años de historia. El segundo, por haber sido eliminada la homosexualidad de las clasificaciones médicas oficiales, como la de la Organización Mundial de Salud en 1992. Precisamente este ítem tuvo mayor sesgo hacia el desacuerdo que la mayoría de los ítems en el estudio original. Los autores no informaron de las propiedades de consistencia interna, estructura factorial y forma de la distribución de la escala original, por lo cual dichas propiedades se calcularon en la presente muestra. Mayor puntuación en la escala HFK10 refleja mayor homofobia externalizada. 


\section{Procedimiento}

Se realizó un estudio descriptivo-correlacional con un diseño ex post-facto transversal. El cuestionario se administró de forma autoaplicada en los salones de clase por los autores del artículo. La aplicación duraba unos 15 minutos; fue realizado de enero a mayo de 2012. Se solicitó, además, el consentimiento informado para la participación en el estudio de forma verbal, garantizándose el anonimato y confidencialidad de la información de acuerdo con las normas éticas de investigación de la Sociedad Mexicana de Psicología (2007).

\section{Análisis de datos}

En relación con los siete objetivos específicos definidos se hicieron los siguientes análisis:

1) Se exploró estructura dimensional por análisis factorial exploratorio, extrayendo los factores por GLS y rotando la matriz de factores por el método Oblimin. El número de factores se determinó por el análisis paralelo de Horn. Se generaron 100 muestras con 20 ó 10 variables normalmente distribuidas con 231 casos aleatorios; ordenadas sus curvas de sedimentación por la pendiente, para definir el punto de intersección con la curva de sedimentación de los datos observados, se tomó la curva correspondiente al percentil 95. El número de autovalores de la curva de sedimentación de los datos observados por encima del punto de intersección definió el número de factores no atribuibles al azar bajo el criterio de Horn. A su vez, para definir el número de factores, se usó el criterio de Kaiser (autovalores mayores que 1) y el de la expectativa (3 factores). Toda carga factorial por debajo de .30 en la matriz de configuraciones se estipuló como baja. El análisis paralelo de Horn se realizó con el programa Vista7.9 (Young, 2010) y el resto de los cálculos del análisis factorial exploratorio con SPSS16 (SPSS Incorporation, 2007).
2) La consistencia interna se estimó por el coeficiente alfa de Cronbach $(\alpha)$. Los valores de consistencia interna menores a .60 se consideraron bajos, de .60 a .69 aceptables y de .70 o mayores altos (Cronbach \& Shavelson, 2004).

3) El contraste de la bondad de ajuste entre el modelo trifactorial y los modelos alternativos se realizó por análisis factorial confirmatorio. La función de discrepancia y parámetros se estimaron por el método GLS. Se optó por este método, al ser robusto a la violación del supuesto de normalidad multivariada (Cragg, 1983) y poder ser aplicado tanto en el análisis exploratorio como confirmatorio. Los valores de la curtosis multivariada de Mardia (90.02) y razón crítica (23.06) fueron altos, indicando incumplimiento del supuesto de normalidad multivariada. Se contemplaron seis índices de ajuste: cociente entre el estadístico chi-cuadrado y sus grados de libertad $\left(\chi^{2} / g l\right)$, valor de la función de discrepancia (FD), parámetro de no centralidad poblacional (PNCP), índice de bondad de ajuste (GFI) de Jöreskog y Sörbom y su modalidad corregida (AGFI) y error cuadrático medio de aproximación (RMSEA) de SteigerLind. Se estipularon como valores de buen ajuste para los índices: $\chi^{2} / g l \leq 2$, FD y PNCP $\leq$ un cuarto de los valores correspondientes al modelo independiente, GFI $\geq .95, \mathrm{AGFI} \geq .90 \mathrm{y}$ RMSEA $\leq .05$; y como valores aceptables: $\chi^{2} / \mathrm{gl}$ $\leq 3$, FD y $\mathrm{PNCP} \leq$ tres cuartos de los valores correspondientes al modelo independiente, GFI $\geq .85$, AGFI $\geq .80$ y RMSEA $\leq .08$. La parsimonia del modelo se estimó desde la razón de parsimonia (RP) de James-Mulaik-Brett (alta $\geq$ .70 , media $\geq .30$ y baja $<.30$ ). La adecuación del tamaño muestral se juzgó desde el tamaño crítico de Hoelter; se consideró muy bueno si el tamaño crítico era mayor que el muestral con un nivel de significación de .01 y bueno con nivel de significación de .05 (Moral, 2006; Kline, 2010). Para comparar la bondad de ajuste de los modelos se empleó la prueba de la diferencia de los estadísticos chi-cuadrado. Con una $p$ 
$>.05$ se mantuvo la hipótesis nula de equivalencia de bondad de ajuste. Todos estos cálculos se realizaron con el programa AMOS16 (Arbuckle, 2007). La potencia de los contrastes por análisis factorial confirmatorio se estimó desde la RMSEA. Se calculó con un nivel de significación de .05. Se empleó como hipótesis nula el valor medio del modelo independiente (mínima constricción) y como hipótesis alternativa el valor medio del modelo especificado. El cálculo se hizo con el programa de Preacher y Coffman (2006).

4) El ajuste de la distribución a una curva normal se contrastó por la prueba de KolmogorovSmirnov $\left(Z_{K-S}\right)$.

5) Las comparaciones de medias entre factores se realizaron por la prueba $t$ de Student para dos muestras emparejadas. De forma previa, el rango de los factores se homogeneizó al dividir la puntuación de cada uno por su número de ítems.

6) Las correlaciones entre las escalas ATLG, HNI-16 y HFK-10 y sus factores se calcularon por el coeficiente producto-momento de Pearson $(r)$. Los valores de correlación menores a .30 se consideraron bajos, de .30 a .69 , moderados y de .70 o mayores altos (Cohen, Cohen, West \& Aiken, 2003).

7) Se realizaron las comparaciones de medias entre hombres y mujeres, entre quienes se definen como heterosexuales y los que no, entre personas que han iniciado su vida sexual de pareja y aquellos que no, quienes tienen amistades homosexuales y aquellos que no, quienes tienen amigos infectados de $\mathrm{VIH}$ y aquellos que no, y entre estudiantes que han atendido a pacientes infectados de $\mathrm{VIH}$ y los que no por medio de la prueba t de Student para muestras independientes. El tamaño del efecto de estas variables dicotómicas sobre la puntuación total de la ATLG y sus factores se estimó por la $d$ de Cohen.

Estos cálculos estadísticos del inciso 2 y del 4 al 7 se ejecutaron con SPSS16.

\section{Resultados}

\section{Exploración de la estructura factorial y con- sistencia interna}

Dos autovalores de la matriz de correlaciones de los 10 ítems de actitud hacia los hombres homosexuales fueron mayores que 1. Por el criterio de Kaiser se definieron dos factores que explicaron $45.13 \%$ de la varianza total. Al rotar la matriz factorial por el método Oblimin se obtuvo un primer factor integrado por 5 ítems G2: "pienso que los hombres homosexuales son repugnantes" ( $I=.99)$, G3: "a los hombres homosexuales no debería permitírseles enseñar en los colegios" ( $I=.55)$, G4: "la homosexualidad masculina es una perversión" ( $/$ = .61), G6: "si un hombre tiene sentimientos homosexuales, debería hacer todo lo posible para superarlos" ( $I=.41)$, y G10: "la homosexualidad masculina es un tipo diferente de opción de vida que no debería ser condenada" ( $I=-.44)$. Con base en el contenido de los mismos se denominó rechazo abierto (ATG-A). El segundo quedó constituido también por 5 ítems: G1: "a las parejas de hombres homosexuales debería permitírseles adoptar hijos como a las parejas heterosexuales" (I = -.88), G5: "la homosexualidad masculina es una expresión natural de la sexualidad masculina" ( $I=-.55)$, G7: "si supiera que mi hijo es homosexual yo no estaría deprimido/a" ( $=-.31)$, G8: "el sexo entre dos hombres no es natural" ( $I=.50)$ y G9: "la idea del matrimonio homosexual me parece ridícula" $(I=.51)$. Debido al contenido de los mismos se denominó rechazo sutil (ATG-S). La correlación entre ambos factores fue moderada $(r=.62, p<$ .01). La consistencia interna de ambos fue alta: .78 para ATG-A y .79 para ATG-S.

Sólo un autovalor de la matriz de correlaciones de los 10 ítems de actitud hacia lesbianas fue mayor que 1. Por el criterio de Kaiser se definió un factor que explicó $45.63 \%$ de la varianza total. Todos los ítems tuvieron cargas mayores que .30, variando de .87 (L3) a .47 (L4), con un promedio de .66. 
Al realizar el análisis paralelo de Horn con los 10 ítems de ATG sólo un autovalor quedó por encima del punto de intersección (1.30), al igual que con los 10 ítems de ATL (1.29). Al aplicarlo a los 20 ítems de la escala ATLG, sólo un autovalor quedó por encima del punto de intersección (1.54). El primer autovalor fue de 8.73 y el segundo 1.34. Al extraer sólo un factor, con base en el criterio de Horn, se explicó 43.66\% de la varianza total y las cargas factoriales variaron de .85 a .47. Al forzar la solución a dos o tres factores, se mezclaron los ítems de actitud hacia hombres homosexuales y lesbianas en la integración de los factores; no se logró la configuración esperada.

La consistencia interna de los 20 ítems fue alta $(\alpha=.91)$, al igual que la de los 10 ítems de actitud hacia las lesbianas $(\alpha=.88)$ y hacia los hombres homosexuales $(\alpha=.86)$.

\section{Contraste de modelos estructurales por análisis factorial confirmatorio}

Se contrastaron tres modelos con parsimonia alta (RP > .70). El tamaño muestral fue muy bueno para el contraste de estos modelos con base en la $N$ crítica de Hoelter y la potencia estimada desde la RMSEA fue alta $(\varphi=.95)$ (véase tabla 1).

En primer lugar, se contrastó el modelo esperado de tres factores jerarquizados a uno general de segundo orden; los tres factores de primer orden coincidieron con los obtenidos en el análisis exploratorio de las subescalas ATG y ATL, al fijar el número de factores por el criterio de Kaiser en cada una de ellas. Todos los parámetros fueron significativos, salvo la varianza residual de la variable latente ATGS. El factor general explicó de 92 a $96 \%$ de la varianza de los tres factores jerarquizados. Los valores de los índices de ajuste de este modelo jerarquizado variaron de buenos $\left(\chi^{2} / \mathrm{gl}=1.72 \mathrm{y}\right.$ RMSEA $=.06$ con IC 90\% [.04, .07], manteniendo la hipótesis nula de RMSEA $\leq .05$ con una $p=.17$ ) a aceptables (FD $=1.25, \mathrm{PNCP}=0.53$,
$\mathrm{GFI}=.87$ y $\mathrm{AGFI}=.84$ ), aunque la bondad de ajuste se rechazó por la prueba chi-cuadrada $\left(\chi^{2}[167, \mathrm{~N}=231]=288.15, p<.01\right)$ (véase figura 1 y tabla 1 ).

En segundo lugar, se contrastó el modelo esperado desde la propuesta de Herek (1984) de dos factores correlacionados con 10 indicadores cada uno. Todos los parámetros fueron significativos. Los índices de ajuste fueron de buenos $\left(\chi^{2} / g l=1.73\right.$ y RMSEA $=.06$ con IC $90 \%[.04, .07]$, manteniendo la hipótesis nula de RMSEA $\leq .05$ con una $p=.16$ ) a aceptables $(\mathrm{FD}=1.27, \mathrm{PNCP}=0.54, \mathrm{GFI}=.87$ y $\mathrm{AGFI}=$ .84), aunque la bondad de ajuste se rechazó también por la prueba chi-cuadrada $\left(\chi^{2}[169, N\right.$ $=231]=293.24, p<.01)$. La correlación entre los dos factores fue muy alta $(r=.94)$ (figura 2 y tabla 1). Por la prueba de la diferencia de los estadísticos chi-cuadrado, la bondad de ajuste del modelo de dos factores correlacionados fue estadísticamente equivalente a la del modelo jerarquizado $\left(\Delta \chi^{2}[2, N=231]=5.09, p=.08\right)$.

En tercer lugar, se contrastó el modelo unifactorial con 20 indicadores; en este estudio es defendido por varios autores (Blackwell \& Kiehl, 2008; Chonody et al., 2011) y sustentado por el análisis factorial del conjunto de ítems desde el criterio de Horn. Todos sus parámetros fueron significativos. La varianza explicada de los ítems fue de 27 a $80 \%$ con un promedio de $49 \%$. Los valores de los índices de ajuste de este modelo unidimensional variaron de buenos $\left(\chi^{2} / \mathrm{gl}=1.81\right.$ y RMSEA $=.06$ con IC 90\% [.05, .07], manteniendo la hipótesis nula de RMSEA $\leq .05$ con una $p=.08$ ) a aceptables (FD = 1.33, $\mathrm{PNCP}=0.60, \mathrm{GFI}=.87$ y $\mathrm{AGFI}=.84$ ), aunque la bondad de ajuste también se rechazó por la prueba chi-cuadrada $\left(\chi^{2}[170, N=231]=307.13\right.$, $p<.01)$. Por la prueba de la diferencia de los estadísticos chi-cuadrado, la bondad de ajuste del modelo de un factor fue estadísticamente menor que la del modelo de dos factores correlacionados $\left(\Delta \chi^{2}[1, N=231]=13.89, p<.01\right)$ y que la del modelo jerarquizado de tres factores $\left(\Delta \chi^{2}[3, N=231]=18.98, p<.01\right)$ (véase figura 3 y tabla 1$)$. 


\section{Tabla 1}

Índices de ajuste para el modelo de un factor, de dos factores correlacionados $y$ de tres factores jerarquizados a uno general por Mínimos Cuadrados Generalizados

\begin{tabular}{|c|c|c|c|c|c|c|}
\hline \multicolumn{2}{|c|}{ Estadísticos } & \multicolumn{2}{|c|}{ Interpretación } & \multicolumn{3}{|c|}{ Modelos } \\
\hline & & Bueno & Malo & $1 \mathrm{~F}$ & $2 \mathrm{~F}$ & $\mathrm{~J}-3 \mathrm{~F}$ \\
\hline \multicolumn{4}{|l|}{$\chi^{2}$} & 307.13 & 293.24 & 288.15 \\
\hline \multicolumn{2}{|l|}{ gl } & & & 170 & 169 & 167 \\
\hline \multicolumn{2}{|l|}{$p$} & $\geq .05$ & $<.01$ & $<.01$ & $<.01$ & $<.01$ \\
\hline \multicolumn{2}{|l|}{$\chi^{2} / g l$} & $\leq 2$ & $>3$ & 1.81 & 1.73 & 1.72 \\
\hline \multicolumn{2}{|l|}{ GFI } & $\geq .95$ & $<.85$ & .87 & .87 & .87 \\
\hline \multicolumn{2}{|l|}{ AGFI } & $\geq .90$ & $<.80$ & .84 & .84 & .84 \\
\hline \multicolumn{2}{|l|}{ FD } & $\leq 0.45$ & $>1.35$ & 1.33 & 1.27 & 1.25 \\
\hline \multicolumn{2}{|l|}{ PNCP } & $\leq 0.24$ & $>0.73$ & 0.60 & 0.54 & 0.53 \\
\hline \multirow{2}{*}{\multicolumn{2}{|c|}{ RMSEA }} & $\leq 0.05$ & $>0.09$ & & & ו7ר \\
\hline & & $p \geq .05$ & $p<.01$ & $\mathrm{p}=.08$ & $p=.16$ & $p=.17$ \\
\hline \multicolumn{2}{|l|}{$\mathrm{RP}$} & $\geq .70$ & $<.30$ & .89 & .89 & .88 \\
\hline \multirow[t]{2}{*}{$\begin{array}{l}\mathrm{N} \\
\text { crítica }\end{array}$} & $\begin{array}{l}\alpha= \\
.05\end{array}$ & & $\mathrm{~N}_{\text {crítica }}>\mathrm{N}$ & 151 & 158 & 159 \\
\hline & $\begin{array}{l}\alpha= \\
.01\end{array}$ & $\mathrm{~N}_{\text {critica }} \leq \mathrm{N}$ & & 162 & 169 & 170 \\
\hline \multicolumn{2}{|l|}{$\varphi$} & $\geq .80$ & $<.60$ & .95 & .95 & .95 \\
\hline
\end{tabular}

Modelos: $1 \mathrm{~F}=$ un factor general, $2 \mathrm{~F}=$ dos factores correlacionados y J.-3F $=$ tres factores jerarquizados a uno general.

Índices de ajuste: $\chi^{2}=$ prueba de bondad de ajuste chi-cuadrado; gl = grados de libertad del estadístico chicuadrado,; $\mathrm{p}=$ probabilidad de mantener la hipótesis nula de bondad de ajuste del estadístico ji-cuadrado obtenido; $\chi^{2} / \mathrm{gl}=$ cociente entre el estadístico chi-cuadrado y sus grados de libertad; FD = valor de la función de discrepancia; GFI = índice de bondad de ajuste; $A G F I=$ índice de bondad de ajuste ajustado de Jöreskog y Sörbom; PNCP = parámetro de no centralidad poblacional(valor medio); y RMSEA = residuo cuadrático medio de aproximación de Steiger-Lind con su valor medio, límites inferior y superior con un intervalo de confianza de $90 \%$ y probabilidad de mantener la hipótesis nula de que RMSEA $\leq .05$.

Parsimonia: RP = razón de parsimonia de James-Mulaik-Brett.

Adecuación del tamaño muestral: Ncrítica= tamaño de muestra crítico de Hoelter con un nivel de significación de .05 .01 para mantener la hipótesis nula de bondad de ajuste con el estadístico ji-cuadrado y grados de libertad obtenidos.

Potencia: $\varphi=$ potencia calculada desde RMSEA.

Valor de la función de discrepancia del modelo independiente $(F D I)=1.80$. Valor medio del parámetro de no centralidad poblacional del modelo independiente $(\mathrm{PNCPI})=0.97$. Valor medio de la RMSEA del modelo independiente $=.08$. 


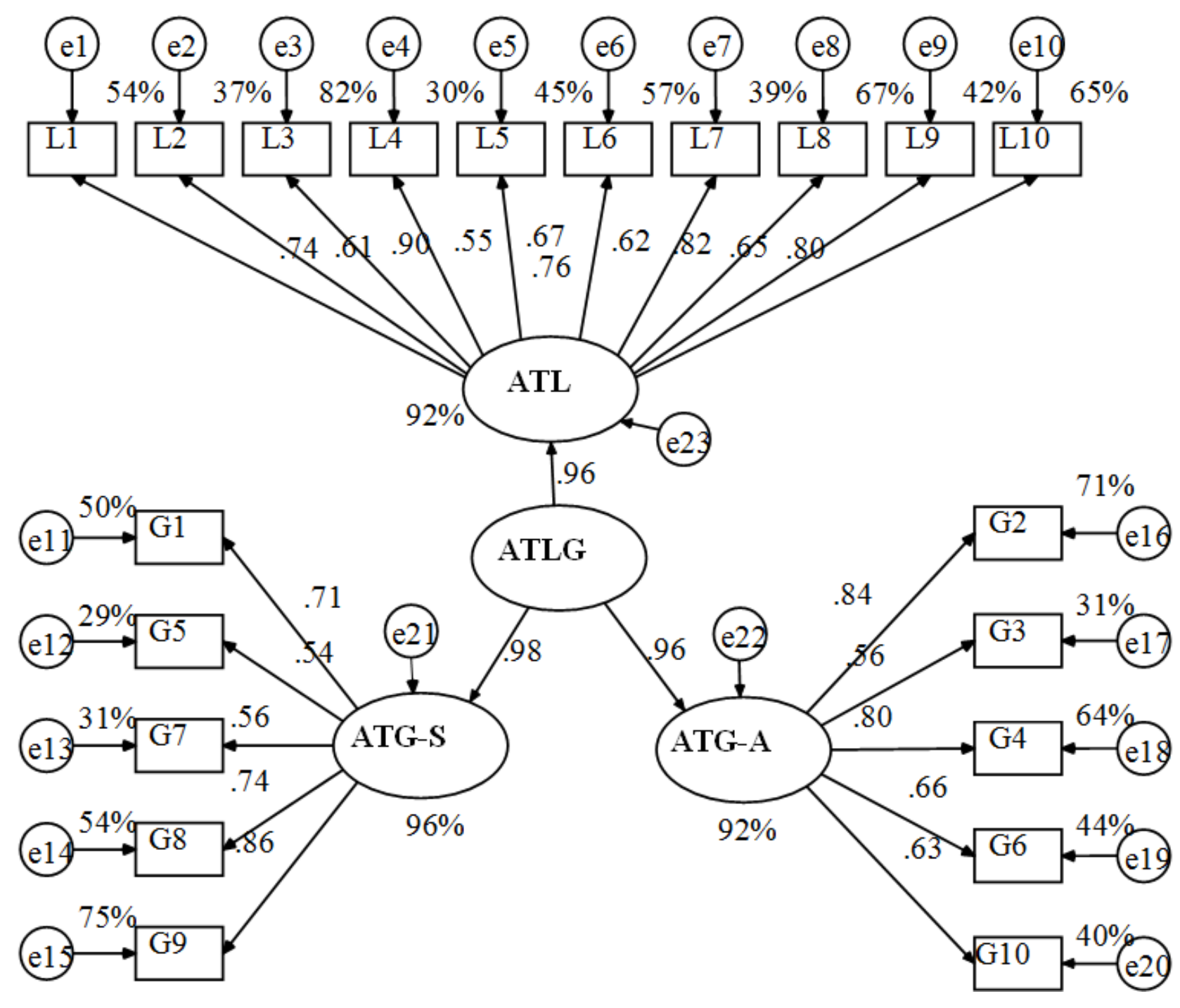

Figura 1. Modelo jerarquizado para ATLG con sus valores estandarizados estimado por Mínimos Cuadrados Generalizados.

Ítems: L1. Las lesbianas no deberían ser integradas en nuestra sociedad. L2. La homosexualidad de una mujer no debería ser una causa de discriminación. L3. La homosexualidad femenina es mala para nuestra sociedad porque rompe la división natural entre los sexos. L4. Las leyes que castigan la conducta sexual consentida por dos mujeres adultas deben ser abolidas. L5. La homosexualidad femenina es un pecado. L6. El número creciente de lesbianas indica una declinación de los valores fundamentales de nuestra sociedad. L7. La homosexualidad femenina por sí misma no es un problema a menos que la sociedad la transforme en un problema. L8. La homosexualidad femenina es una amenaza para muchas de nuestras instituciones sociales básicas como la familia. L9. La homosexualidad es una forma inferior de sexualidad. L10. Las lesbianas son enfermas. G1. A las parejas de hombres homosexuales debería permitírseles adoptar hijos como a las parejas heterosexuales. G2. Pienso que los hombres homosexuales son repugnantes. G3. A los hombres homosexuales no debería permitírseles enseñar en los colegios. G4. La homosexualidad masculina es una perversión. G5. La homosexualidad masculina es una expresión natural de la sexualidad masculina. G6. Si un hombre tiene sentimientos homosexuales, debería hacer todo lo posible para superarlos. G7. Si supiera que mi hijo es homosexual yo no estaría deprimido/a. G8. El sexo entre dos hombres no es natural. G9. La idea del matrimonio homosexual me parece ridícula. G10. La homosexualidad masculina es un tipo diferente de opción de vida que no debería ser condenada.

ATLG: Rechazo hacia lesbianas y hombres homosexuales. ATL = Rechazo hacia las lesbianas. ATG-A = Rechazo abierto hacia los hombres homosexuales. ATG-S = Rechazo sutil hacia los hombres homosexuales. 
Distribución y diferencias de medias entre los factores en las comparaciones intra-participantes

La puntuación total de la escala ATLG se ajustó a una curva normal $\left(Z_{K-S}=1.01, p=.26\right)$ con media de 70.75 y error estándar de 1.89 (95\% IC: $67.03,74.47)$. También la subescala ATG se ajustó a una curva normal $\left(Z_{K-S}=1.14\right.$, $p=.15)$ con media de 39.62 y error estándar de
1.01 (95\% IC: $37.63,41.61)$ y la subescala ATL $\left(Z_{k-S}=1.16, p=.13\right)$ con media de 31.13 y error estándar de 0.97 (95\% IC: 29.22, 33.05). Al separar los ítems de rechazo hacia los hombres homosexuales en dos factores, la distribución de rechazo sutil (ATG-S) sí se ajustó a una curva normal $\left(Z_{K-S}=0.93, p=.36\right)$ con media de 24.77 y error estándar de 0.62 (95\% IC: 23.54 , 25.99), pero la distribución de rechazo abierto (ATG-A) no se ajustó a una curva normal $\left(Z_{k-S}=\right.$

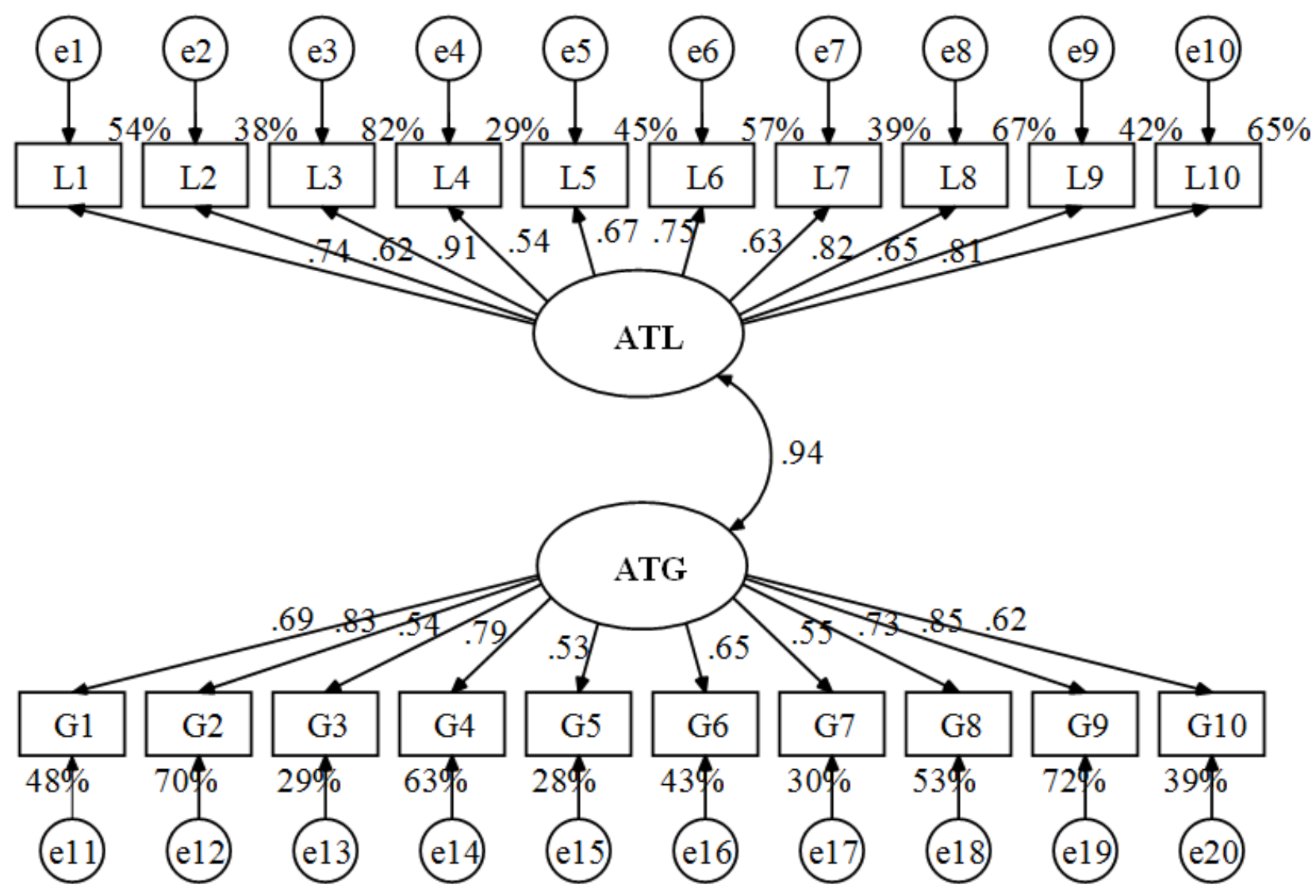

Figura 2. Modelo de dos factores correlacionados para ATLG con sus valores estandarizados estimado por Mínimos Cuadrados Generalizados.

ATL $=$ Rechazo hacia las lesbianas. ATG $=$ Rechazo hacia los hombres homosexuales. 
$1.60, p=.01$ ), siendo su media de 14.85 y error estándar de 0.49 (95\% IC: 13.89, 15.82).

Se dividió la media de cada escala por el número de ítems que la integraban para interpretar la tendencia actitudinal en un rango de 1: "definitivamente en desacuerdo" a 9: "definitivamente de acuerdo" desde una redacción de rechazo. Siendo el valor mínimo 1 y el máximo 9 entre 5 valores discretos, el intervalo de incremento sería 1.6 ([valor máximo - valor mínimo]/ número de intervalos $=[9-1] / 5)$. Los valores de
1 a 2.59 (valor discreto 1) reflejan una actitud de definitivamente en desacuerdo con el rechazo, de 2.60 a 4.19 (valor discreto 3) en desacuerdo, de 4.20 a 5.79 (valor discreto 5) indiferente, de 5.80 a 7.39 (valor discreto 7 ) de acuerdo, y de 7.40 a 9 (valor discreto 9) definitivamente de acuerdo. Las medias de ATG-A (2.97), ATL (3.11), ATLG (3.54) y ATG (3.96) correspondieron al intervalo del valor discreto 3 (en desacuerdo) y la media de ATG-S (4.95) correspondió al intervalo del valor discreto 5 (indiferente).

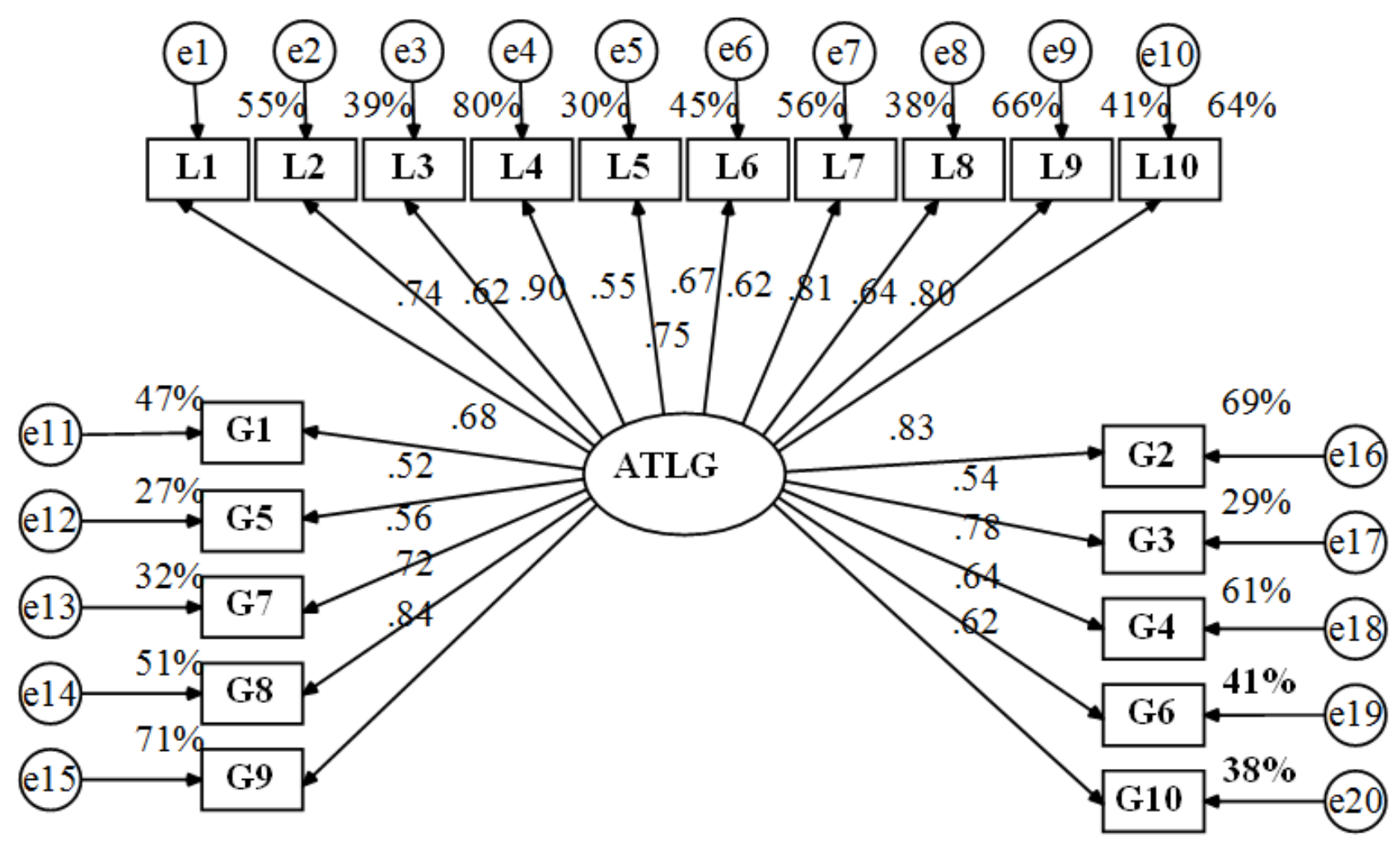

Figura 3. Modelo unidimensional para ATLG con sus valores estandarizados estimado por Mínimos Cuadrados Generalizados 
La media del rechazo hacia los hombres homosexuales fue significativamente mayor que la media de rechazo hacia las lesbianas ( $t[230]$ $=13.84, p<.01$ ) en las comparaciones intraparticipantes. La media del rechazo sutil hacia los hombres homosexuales fue significativamente mayor que el rechazo abierto hacia los hombres homosexuales $(t[230]=20.69, p<$ $.01)$ y hacia las lesbianas $(t[230]=24.23, p<$ .01). El rechazo abierto hacia los hombres homosexuales fue mayor que el rechazo hacia las lesbianas $(t[230]=9.23, p<.01)$.

\section{Validez convergente con homonegatividad internalizada y homofobia externalizada}

En primer lugar, se estudiaron las propiedades psicométricas de la escala HFK-10, al ser éstas totalmente desconocidas. La consistencia interna de los 10 ítems de la escala HFK10 fue alta $(\alpha=.84)$. Por el análisis paralelo de Horn, el número de factores fue uno. Al ser extraído este factor por análisis de componentes principales se explicó $42 \%$ de la varianza total. Las cargas factoriales en este componente único variaron de .44 a .79, con una media de .64 . Al contrastar un modelo unidimensional con 10 indicadores por GLS el ajuste fue aceptable: $\chi^{2} / \mathrm{gl}=2.74, \mathrm{GFI}=.93, \mathrm{AGFI}=.87, \mathrm{FD}=0.42$, $\mathrm{PNCP}=0.26$ y $\mathrm{RMSEA}=.09$.

La puntuación total de la escala ATLG tuvo correlaciones significativas, directas y altas con la puntuación total de la HNI-16 $(r=.72, p<.01)$ y de la HFK-10 ( $r=.79, p<.01)$. La homonegatividad internalizada correlacionó significativamente más alto $(t[228]=2.62, p<.01)$ con el rechazo sutil hacia los hombres homosexuales $(r=.70, p<.01)$ que con el rechazo abierto $(r=$ $.60, p<.01)$; por el contrario, la homofobia externalizada correlacionó significativamente más alto $(t[228]=2.03, p=.02)$ con el factor de rechazo abierto hacia los hombres homosexuales $(r=.74, p<.01)$ que con el rechazo sutil $(r=.67$, $p<.01$ ) (véase tabla 2).

\section{Validez de constructo: diferencias de me- dias entre grupos independientes}

Respecto a la orientación sexual autodefinida, 95\% (220 de 231) se definió heterosexual, $3 \%$ (7) bisexual y $2 \%$ (4) homosexual. Ante la pregunta sobre si habían iniciado su vida sexual de pareja, 62\% (142 de 230) dijo que no y $38 \%$ (88), que sí. El promedio de parejas sexuales entre estas 88 personas fue de 3.11 (DE = 5.86) con un mínimo de 1 y máximo de 50 . Ante la pregunta si tenían amigos homosexuales, $75.5 \%$ (173 de 229) indicó que sí y 24.5\% (56), que no; y si tenían amigos infectados de $\mathrm{VIH}$, $98 \%$ (222 de 227) señaló que no y 5 (2\%) que sí. De los 227 encuestados que respondieron a la pregunta de si habían atendido a pacientes infectados de VIH, $88 \%$ (199) dijo que no y $12 \%$ (28), que sí.

Se halló diferencia significativa de medias entre los dos grupos de orientación sexual autodefinida y tener amigos homosexuales. El tamaño del efecto de ambas variables fue me-

Tabla 2

Correlaciones de ATLG con HNI-16 y HFK-10

\begin{tabular}{ccc}
\hline Actitudes & $\begin{array}{c}\text { Homofobia } \\
\text { externalizada (HFK-10) }\end{array}$ & $\begin{array}{c}\text { Homonegatividad } \\
\text { internalizada (HNI-16) }\end{array}$ \\
\hline ATLG & $.79^{* *}$ & $.72^{\star *}$ \\
ATL & $.73^{\star *}$ & $.65^{\star \star}$ \\
ATG & $.77^{\star *}$ & $.72^{\star *}$ \\
ATG-A & $.74^{* *}$ & $.60^{\star *}$ \\
ATG-S & $.67^{\star *}$ & $.70^{\star *}$ \\
\hline
\end{tabular}

$N=312 .{ }^{* *} p<.01$. ATLG: Rechazo hacia lesbianas y hombres homosexuales. ATG = Rechazo hacia los hombres homosexuales; ATG-A = Rechazo abierto hacia los hombres homosexuales, ATG-S = Rechazo sutil hacia los hombres homosexuales, ATL = Rechazo hacia las lesbianas. 
diano sobre ATLG, ATG y ATG-S; el tamaño del efecto de heterosexualidad fue grande sobre ATL y pequeño sobre ATG-A; y el de amistades homosexuales fue mediano sobre ATG-A y bajo en ATL. Quienes se definieron como hete- rosexuales y quienes dijeron no tener amigos homosexuales mostraron mayor rechazo. Las medias de los que tenían amigos infectados de $\mathrm{VIH}$ fueron menores de aquellos que no en los factores ATG y ATG-A con un tamaño de efecto pequeño (véase tabla 3 ).

Tabla 3

Comparación de medias por la prueba t de Student para dos muestras independientes

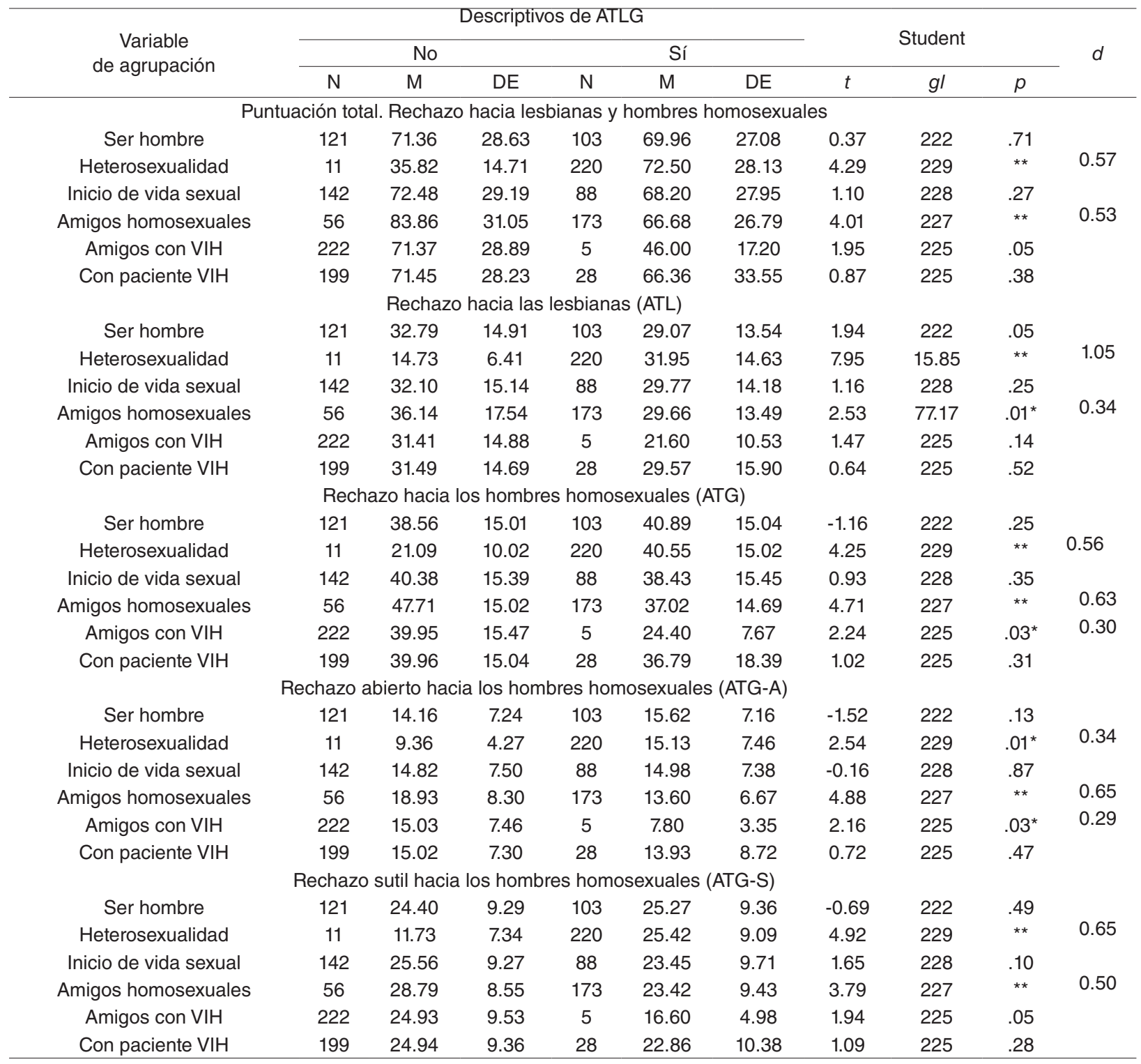

${ }^{* *} p<.01,{ }^{*} p<.05 . d$ de Cohen: $2 t /(n 1+n 2-2) 1 / 2$ para estimar el tamaño del efecto. 


\section{Discusión}

Los presentes datos apoyan de forma clara un modelo unidimensional, como es defendido por autores como Chonody et al. (2011) y Blackwell y Kiehl (2008). El análisis paralelo de Horn indica que más de un factor sería artificioso; la configuración de un segundo o tercer factor se pudiera deber al azar. Al estimar los parámetros de modelos de dos factores, las correlaciones entre ambos factores fueron tan altas que deberían considerarse como uno. El modelo jerarquizado lo expresa de forma muy explícita. Los coeficientes de determinación de los tres factores jerarquizados son casi unitarios. Con cualquiera de ellos tres se podría medir la variable latente. Incluso el factor de rechazo sutil hacia los hombres homosexuales queda totalmente explicado por el factor general y carece de varianza residual. Además, la consistencia interna de los 20 ítems es mayor que .90, lo que es propio de escalas unidimensionales (Cronbach \& Shavelson, 2004).

El modelo jerarquizado y el de dos factores correlacionados traen unos índices de ajuste equivalentes y mejores que el unidimensional, pero finalmente las diferencias de ajuste son mínimas; asimismo, la parsimonia y potencia son equivalentes entre los modelos. Todo lo anterior inclina los argumentos a favor del modelo de un factor con 20 indicadores.

Se podría esperar que el patrón de respuesta a los ítems fuese más homogéneo en los aspectos de rechazo manifiesto (actitud clara de desacuerdo colectivo) y más heterogéneo en los aspectos de rechazo sutil (menor consenso social y mayor variabilidad individual). No obstante, los valores de consistencia son muy semejantes entre los dos factores de 5 ítems de actitud hacia los hombres homosexuales y en un rango que se interpreta como alto. Una diferencia aparente entre los factores es la mayor consistencia que presentaron los 10 ítems de actitud hacia las lesbianas. Esto se debe a su mayor número de ítems (Cronbach \& Shavel- son, 2004), de modo que, al calcular la consistencia interna con los 10 ítems de actitud hacia los hombres homosexuales, ésta se asemeja mucho (.86 versus .88). Por lo tanto, al igual que con las correlaciones, la consistencia interna refleja que estos factores son equiparables y sustituibles unos por otros.

Las medias de la puntuación total y los distintos factores fueron diferenciales entre los dos grupos de orientación sexual autodefinida (heterosexual o no) y tener amistades homosexuales (sí o no), siendo su tamaño de efecto moderado en la mayoría de las comparaciones, lo que apunta hacia lo intercambiable que es un factor por otro.

No obstante, sí se observan algunas diferencias en los patrones de correlaciones y de diferencias de medias. La actitud de rechazo sutil hacia los hombres homosexuales se correlaciona más con homonegatividad internalizada que con homofobia externalizada; a la inversa, la actitud de rechazo abierto hacia los hombres homosexuales se correlaciona más con homofobia externalizada que con homonegatividad internalizada. Este patrón diferencial se comporta conforme a lo esperado por el contenido de los dos factores de actitud y de las escalas HFK-10 y HNI-16. Otra diferencia se presenta en las medias de la actitud hacia los hombres homosexuales entre los dos grupos de participantes que tienen o no amigos infectados de VIH. La diferencia sí es significativa en rechazo abierto, pero no en el rechazo sutil. Así la experiencia con el objeto estigmatizado por la ideología heterosexista tiende a modificar la expresión de un rechazo abierto, pero no a un nivel más profundo de rechazo sutil o simbólico. Estas pocas diferencias en correlaciones y comparaciones de medias frente a las muchas semejanzas tienen un tamaño de efecto pequeño, no constituyen finalmente argumentos fuertes a favor del modelo trifactorial jerarquizado.

Otra diferencia surge a nivel de interpretación (aceptación-rechazo) de los promedios. El factor de rechazo sutil hacia los hombres homo- 
sexuales presenta la media más alta, lo que significa que elicita mayor rechazo. En este factor, la muestra de estudiantes presenta una actitud ambigua entre el rechazo y la aceptación cuando en los otros factores la actitud es de desacuerdo con el rechazo hacia los hombres homosexuales y las lesbianas. Esto conforme a las expectativas en función de los cambios actuales en la cultura occidental. Hay una aceptación creciente de la diversidad sexual, no se criminaliza la homosexualidad, sino que se consideran un delito los ataques y la discriminación hacia personas homosexuales, pero persiste el rechazo que se manifiesta en formas sutiles y simbólicas de valoración y trato diferencial (Herek, 2004).

Si se establece que el factor de rechazo sutil hacia los hombres homosexuales tiene una consistencia interna alta, carece de varianza residual al ser explicado totalmente por el factor general de actitud hacia la homosexualidad, posee el mayor promedio en comparación con los otros factores e incluso la puntuación total. Esto último evidencia que el rechazo implícito es mayor que el explícito, entonces se podría utilizar como una forma simplificada de la escala, especialmente en estudios que empleen un número muy grande de instrumentos y requieran reducir el número de ítems.

Como en otros estudios (Herek \& McLemore, 2013), en éste se observa un mayor rechazo hacia los hombres homosexuales que hacia las lesbianas, situación acorde con los valores judeocristianos que predominan en la sociedad mexicana, los cuales condenan con mayor severidad la desviación homosexual en el hombre que en la mujer (Wittig, 2005). Debe señalarse que el control social de la sexualidad femenina va dirigido más hacia el número y concurrencia de parejas que hacia la orientación sexual (Díaz-Guerrero, 2003; Vázquez \& Chávez, 2008).

La actitud es equivalente entre hombres y mujeres en el puntaje total y en la actitud hacia los hombres homosexuales. En la actitud hacia las lesbianas, la diferencia se aproxima a la significación estadística, siendo mayor el promedio de las mujeres, como se esperaba a causa de la función expresiva de la actitud en defensa de una ideología heterosexista, al rechazar más la homosexualidad en el propio sexo. También los promedios de los hombres son más altos que los de las mujeres en el rechazo hacia los hombres homosexuales, aunque la diferencia no es estadísticamente significativa por la amplia variabilidad dentro de cada sexo. Esta gran semejanza en la actitud de ambos sexos, junto con porcentajes bajos de rechazo $(8 \%)$ e indiferencia (22\%) en comparación con el de aceptación (70\%), hablan de un cambio de actitud en los estudiantes actuales frente a los de décadas anteriores, cuando los porcentajes de rechazo y la diferencia entre ambos sexos eran mayores (Herek, 2004).

Se confirmó la expectativa de mayor aceptación entre quienes se definieron como no heterosexuales, tenían amistades homosexuales o infectadas de VIH. Herek y McLemore (2013) argumentan que la pertenencia grupal y el contacto directo con el objeto estigmatizado llevan hacia una posición crítica o de resistencia a la ideología dominante, en este caso el heterosexismo, lo que podría dar cuenta de esta diferencia.

La expectativa era de menor rechazo entre quienes reportaran haber trabajado con pacientes infectados de VIH, al ser la mayoría de los pacientes hombres con prácticas homosexuales (CENSIDA, 2012). Esto implica contacto con el grupo estigmatizado, de ahí que se esperara menor rechazo hacia la homosexualidad, especialmente a mayor tiempo de exposición (Sánchez, Rabatin, Sánchez, Hubbard, \& Kalet, 2006). Los promedios confirmaron la expectativa, pero la diferencia de medias no fue estadísticamente significativa. Esto, en parte, podría deberse a la desigualdad de los tamaños de cada grupo; además, al ser los participantes estudiantes de los primeros semestres, su experiencia clínica era escasa (menor tiempo de 
exposición). La suma de estas dos circunstancias probablemente hizo que la diferencia fuese pequeña y no significativa.

Este estudio tiene como limitaciones: un muestreo no probabilístico, habiéndose realizado en una muestra incidental de estudiantes de Ciencias de la Salud procedentes de tres universidades del noreste de México, por lo cual las conclusiones son aplicables a modo de hipótesis en esta población y otras afines; además, la investigación se redujo a instrumentos de autoinforme, de modo que los resultados podrían diferir con pruebas proyectivas o de tiempos de reacción.

En conclusión, en esta muestra de estudiantes universitarios de Ciencias de la Salud, la estructura factorial de la ATLG resulta unidimensional, son muy limitados los argumentos a favor de un modelo tridimensional con un factor general. No obstante, desde el modelo jerarquizado se puede recomendar el uso del factor de 5 ítems de rechazo sutil hacia los hombres homosexuales si se desea simplificar al mínimo el número de ítems. Los datos de las correlaciones con homofobia externalizada y homonegatividad internalizada, las diferencias de medias y correlaciones entre los factores, así como las diferencias entre grupos de sexos, orientación sexual autodefinida y tipo de amistades apoyan la validez de la escala ATLG. De este modo, se recomienda su uso para la evaluación de la actitud hacia los hombres homosexuales y las lesbianas en estudiantes de Ciencias de la Salud mexicanos. Se sugiere su manejo como una medida unidimensional del constructo. En apoyo al modelo tridimensional se requieren investigaciones adicionales, pues este estudio no lo sustenta, mostrando que es algo forzado o artificial.

Los presentes datos muestran que los estudiantes de Ciencias de la Salud son tolerantes hacia la homosexualidad y rechazan su condena abierta, aunque persisten formas sutiles de rechazo que muestran finalmente, ambigüedad en su actitud; de ahí que se sugiere persistir en las estrategias de fomento de valores de tolerancia y respeto hacia la diversidad sexual en su formación. Esto es un primer paso para hacer frente a los prejuicios, a menudo encubiertos, en profesionales de la salud que van contra una atención ética y de calidad para los pacientes.

\section{Referencias}

Aguirre, J. J., \& Rendón, A. E. (2008). Aproximación a una masculinidad estigmatizada: hombres que tienen sexo con otros hombres. México, DF: CONAPRED.

Arbuckle, J. L. (2007). AMOS 16.0 user's guide. Spring House, PA: Amos Development.

Barrientos, J., \& Cárdenas, M. (2012). A confirmatory factor analysis of the Spanish language version of the Attitudes Toward Lesbians and Gay Men Scale (ATLG). Universitas Psichologica, 11, 579-586.

Blackwell, C. W., \& Kiehl, E. M. (2008). Homophobia in registered nurses: Impact on LGB youth. Journal of LGBT Youth, 5(4), 28-44. http://dx.d oi:10.1080/19361650802222989

Cárdenas, M., \& Barrientos, J. (2008a). The Attitudes Toward Lesbians and Gay Men Scale (ATLG): Adaptation and testing the reliability and validity in Chile. Journal of Sex Research, 45(2), 140-149. doi:10.1080/00224490801987424

Cárdenas, M., \& Barrientos, J. (2008b). Actitudes implícitas y explícitas hacia los hombres homosexuales en una muestra de estudiantes universitarios en Chile. Psykhe, 17(2), 17-25.

Centro Nacional para la Prevención y el Control del SIDA (2012). VIHI SIDA en México 2012. México, DF: CENSIDA.

Chonody, J., Siebert, C. F., Siebert, D. C., \& Rutledget, S. E. (2011). The importance of confirmatory validation: A study of the short version of the attitudes toward lesbians and gays (ATLG-S). Presentation for the annual meeting of the society for social work and research in Tampa, Florida. Jannuary, 2011.

Cohen, J., Cohen, P., West, S. G., \& Aiken, L. S. (2003). Applied multiple regression/correlation analysis for the behavioral sciences (3rd ed.). Mahwah, NJ: Psychology Press.

Córdova, J. A., Ponce, S., \& Valdespino, J. L. (2009). 25 años de SIDA en México. Retos, logros y desaciertos. México, DF: CENSIDA.

Cragg, J. G. (1983). More efficient estimation in the presence of heteroscedasticity of unknown form. Econometrica, 51, 751-764. http:// dx.doi.org/10.2307/1912156

Crompton, L. (2006). Homosexuality and civilization. Cambridge: Belknap.

Cronbach, L. J., \& Shavelson, R. J. (2004). My current thoughts on coefficient alpha and successor procedures. Educational and Psychological Measurement, 64, 91-418. http://dx.doi. org/10.1177/0013164404266386

Currie, M. R., Cunningham, E. G., \& Findlay, B. M. (2004). The Short Internalized Homonegativity Scale: Examination of the factorial structure of a new measure of internalized homophobia. Educational and Psychological Measurement, 64, 1053-1067. http://dx.doi. org/10.1177/0013164404264845

Díaz-Guerrero, R. (2003). Bajo las garras de la cultura. México, DF: Editorial Trillas.

Haddock, G. (2004). Contemporary perspectives on the psychology of attitudes. New York: Publisher Taylor \& Francis.

Herek, G. M. (1984). Attitudes toward lesbians and gay men: A factor analytic study. Journal of Homosexuality, 10(1/2), 39-51. http://dx.doi. org/10.1300/J082v10n01_03 
Herek, G. M. (1994). Assessing heterosexuals' attitudes toward lesbians and gay men: A review of empirical research with the ATLG scale. En B. Greene \& G.M. Herek (Eds.), Lesbian and gay psychology: Theory, research, and clinical applications (pp. 206-228). Thousand Oaks, CA: Sage Publications.

Herek, G. M. (2004). Beyond "homophobia": Thinking about sexual prejudice and stigma in the twenty-first century. Sexuality Research \& Social Policy, 1(2), 6-24. http://dx.doi.org/10.1525/srsp.2004.1.2.6

Herek, G. M. (2009). Hate crimes and stigma-related experiences among sexual minority adults in the United States: Prevalence estimates from a national probability sample. Journal of Interpersonal Violence, 24, 54-74. http://dx.doi.org/10.1177/0886260508316477

Herek, G. M., Chopp, R., \& Strohl, D. (2007). Sexual stigma: Putting sexual minority health issues in context. En I. Meyer \& M. Northridge (Eds.), The health of sexual minorities: Public health perspectives on lesbian, gay, bisexual, and transgender populations (pp. 171-208). New York: Springer. http://dx.doi.org/10.1007/978-0-387-31334-4_8

Herek, G. M., Gillis, J. R., \& Cogan, J. C. (2009). Internalized stigma among sexual minority adults: Insights from a social psychological perspective. Journal of Counseling Psychology, 56, 32-43. http:// dx.doi.org/10.1037/a0014672

Herek, G. M., \& McLemore, K. A. (2011). The Attitudes Toward Lesbians and Gay Men (ATLG) scale. En T. Fisher, C. M. Davis, W. L. Yarber \& S. L. Davis (Eds.), Handbook of sexuality-related measures (3rd ed., pp. 415-417). Oxford, England: Taylor \& Francis.

Herek, G. M., \& McLemore, K. A. (2013). Sexual prejudice. Annual Review of Psychology, 64, 309-333. http://dx.doi.org/10.1146/annurevpsych-113011-143826

Klamen, D. L., Grossman, L. S., \& Kopacz, D. R. (1999). Medical student homophobia. Journal of Homosexuality, 37, 53-63. http://dx.doi. org/10.1300/J082v37n01_04

Kline, R. B. (2010). Principles and practice of structural equation modeling (3rd ed.). New York: The Guilford Press.

Majied, K. (2008). A conceptual analysis of homophobia and heterosexism: Experiences of lesbians, gay, bisexual, transgendered and questioning people (LGBTQ) in Trinidad. The Caribbean Journal of Social Work, 6-7, 144-166.

Major, B., \& Eccleston, C. P. (2005). Stigma and social exclusion. En D. Abrams, J. Marques \& M. A. Hogg (Eds.), Social psychology of inclusion and exclusion (pp. 63-87). New York: Psychol. Press.

Mayer, K. H., Bekker, L. G., Stall, R., Grulich, A. E., Colfax, G., \& Lama, J. R. (2012). Comprehensive clinical care for men who have sex with men: an integrated approach. Lancet, 380(9839), 378-87. http:// dx.doi.org/10.1016/S0140-6736(12)60835-6.

Moral, J. (2006). Análisis factorial confirmatorio. En R. Landero \& M. T. González (Eds.), Estadística con SPSS y metodología de la investigación (pp. 445-528). México, DF: Editorial Trillas.

Moral, J. (2010). Una propuesta explicativa integradora de la conducta homosexual. Revista Electrónica de Motivación y Emoción, 13(34), 1-15. Recuperado de: http://reme.uji.es/articulos/numero34/article9/ texto.html

Moral, J., \& Segovia, P. (2011). Discriminación en mujeres que viven con VIH/SIDA. Revista Iberoamericana de Psicolog ía y Salud.2(2), 139159.

Moral, J., \& Valle, A. (2011). Escala de Actitudes hacia Lesbianas y Hombres Homosexuales en México 1. Estructura factorial y consistencia interna. Revista Electrónica Nova Scientia, 3(2), 139-157.

Moral, J., \& Valle, A. (2012). Escala de Actitudes hacia Lesbianas y Hombres Homosexuales (ATLG) 2. Distribución y evidencias de validez. Revista Electrónica Nova Scientia, 4(1), 153-171.

Moral, J., \& Valle, A. (2013). Dimensionalidad, consistencia interna y distribución de la Escala Homonegatividad Internalizada en estudiantes mexicanos de Ciencias de la Salud. Acta Mexicana de Psicología, 3(1), 22-50.

Neumann, R., Hülsenbeck, K., \& Seibt, B. (2004). Attitudes towards people with AIDS and avoidance behavior: Automatic and reflective bases of behavior. Journal of Experimental Social Psychology, 40, 543-550. http://dx.doi.org/10.1016/j.jesp.2003.10.006
Organización Mundial de la Salud (1992). CIE-10. Trastornos mentales y del comportamiento. Descripciones clínicas y pautas para el diagnóstico. Madrid: Meditor.

Preacher, K. J., \& Coffman, D. L. (2006). Computing power and minimum sample size for RMSEA [Computer software]. Recuperado de: http:// quantpsy.org/rmsea/rmsea.htm

Sánchez, N. F., Rabatin, J., Sánchez, J. P., Hubbard, S., \& Kalet, A. (2006). Medical students' ability to care for lesbian, gay, bisexual, and transgendered patients. Family Medicine, 38, 21-27.

Sequeira, G. M., Chakraborti, C., \& Panunti, B. A. (2012). Integrating lesbians, gay, bisexual and transgender (LGBT) content into undergraduate medical school curricula: A qualitative study. The Ochner Journal, 12(4), 379-382.

Sociedad Mexicana de Psicología. (2007). Código ético del psicólogo (4aㅡ edición). México, DF: Editorial Trillas.

SPSS Incorporation (2007). The SPSS base 16.0. User's guide. Chicago, IL: SPSS Inc.

Stoever, C. J., \& Morera, O. F. (2007). A confirmatory factor analysis of the Attitudes Toward Lesbians and Gay Men (ATLG) measure. Journal of Homosexuality, 52(3-4), 189-209, available via: http://dx.doi:10.1300/ J082v52n03_09

Turner, J. C. ,\& Reynolds, K. (2007). A self-categorization theory. En P.A. M. Van Lange, A. W. Kruglanski \& E. T. Higgins (Eds.), Handbook of theories of social psychology (vol. 2, pp. 399-418). Thousand Oaks, CA: SAGE Publications Ltd. http://dx.doi.org/10.4135/9781446249222. n46

Vázquez, V., \& Chávez, M. E. (2008). Género, sexualidad y el poder. El chisme en la vida estudiantil de la Universidad Autónoma de Chapingo, México. Estudios sobre las Culturas Contemporáneas, 14(27), 77-112.

Weber-Gilmore, G., Rose, S., \& Rubinstein, R. (2011). The impact of internalized homophobia on outness for lesbian, gay, and bisexual individuals. The Professional Counselor: Research and Practice, 1(3), 163-175.

White, M. E., \& Murrell, A. R. (2012). Attitudes and internalized homophobia in gay and lesbian college students. En Association for Contextual Behavior Science (Ed.), Proceedings of $X$ annual world conference. Washington, DC: Author.

Wittig, M. (2005). El pensamiento heterosexual y otros ensayos. Madrid: Egales.

Young, F. W. (2010). ViSta "The Visual Statistics System", version 7.9.2.5 [computer software] [on-line]. Recuperado de: http://www.visualstats.org. 